\title{
Aerodynamic Sensing for a Fixed Wing UAS Operating at High Angles of Attack
}

\author{
Derrick Yeo ${ }^{1}$, Ella M. Atkins. ${ }^{2}$, Luis P. Bernal ${ }^{3}$ \\ Department of Aerospace Engineering, University of Michigan, Ann Arbor, Michigan, 48109, U.S.A \\ and \\ Wei Shyy. ${ }^{4}$ \\ Department of Mechanical Engineering, Hong Kong University of Science and Technology, Kowloon, Hong \\ Kong
}

The flight profile of typical small unmanned air systems (UAS) has expanded in the last decade beyond those encountered by traditional fixed-wing transport and rotary-wing aircraft. These new flight conditions may involve unusual flight mechanics as with flapping wing flight or complex aerodynamics when performing difficult maneuvers as part of routine operations. Such scenarios typically involve unsteady and inherently non-linear aerodynamics that challenge current autopilots relying primarily on inertial measurements and GPS data. The addition of aerodynamic feedback to the current UAS sensing and control framework can enable the autopilots of aircraft operating under such conditions to better achieve their control objectives and expand their autonomous flight capabilities. This paper describes the ongoing development of a pressurebased aerodynamic data system for a fixed wing UAS operating at high angles of attack. Expansions to the aerodynamic instrumentation package based on flight test results are described. These include an expanded set of wing pressure ports and a custom designed multi-hole probe for prop-wash estimation. Autonomous flight test data from the first generation UAS system transitioning between cruise and hover are presented and discussed. Open loop flight data acquired using the upgraded sensing concept is then presented. Flight test results show that the sensing package provides data that is complementary to a typical Inertial Measurement Unit and can support the development of feedback control strategies based on aerodynamic measurements.

$$
\begin{array}{ll}
\mathrm{C}_{\mathrm{p}} & =\text { Coeficent of Pressure } \\
\propto & =\text { Angle of Attack } \\
\beta & =\text { Sideslip Angle } \\
S & =\text { Wing Area } \\
L & =\text { Chord Length } \\
\mathrm{v} & =\text { Viscocity of Air } \\
\mathrm{V}_{\infty} & =\text { Air Speed } \\
\mathrm{K}_{\alpha, \beta \mathrm{i}} & =\text { Polynomial Curve Coefficient } \\
\mathrm{P}_{i} & =\text { Wing Pressure Port Measurement } \\
\mathrm{q} & =\text { Dynamic Pressure Measurement }
\end{array}
$$

Nomenclature

\footnotetext{
${ }^{1} \mathrm{PhD}$ student, Aerospace Engineering Dept, University of Michigan, Ann Arbor, MI, AIAA Student Member

${ }^{2}$ Associate Professor, Aerospace Engineering Dept, University of Michigan, Ann Arbor, MI, Associate Fellow

${ }^{3}$ Associate Professor, Aerospace Engineering Dept, University of Michigan, Ann Arbor, MI, AIAA Member

${ }^{4}$ Provost \& Chair Professor, Department of Mechanical Engineering, Hong Kong University of Science and Technology, also; Adjunct Professor, Department of Aerospace Engineering, University of Michigan, AIAA Fellow
} 


\section{Introduction}

$\mathrm{M}_{\mathrm{r}}^{\mathrm{o}}$ odern autopilots have capably flown a variety of unmanned and manned aircraft in numerous applications. Fixed and rotary wing autopilots have been developed [1,2,3], but most feedback control approaches, particularly for fixed-wing configurations, focus on aerodynamic coefficients linearized about set points within the traditional (attached flow) flight envelope, and inertial instrumentation with a limited complement of air data, most typically airspeed and altitude potentially supplemented by angle of attack and sideslip measurements. Small fixedwing unmanned aircraft systems (UAS) that can operate at high angles of attack can serve a range of mission profiles beyond those commonly associated with fixed wing vehicles. Being able to fly at low airspeeds can allow a degree of flexibility when fielding payloads such as surveillance or scientific survey equipment. A hovering fixed wing UAS that can fly at zero groundspeed can offer a blend of close-range surveillance capabilities and rapid redeployment on a largely conventional airframe. Taking the concept a step further, a perching fixed wing UAS can provide short spot-landing capabilities without the speed disadvantages of a rotary wing vehicle during relocation phases of the mission.

When operating near or outside the flight envelope boundary, a requirement for hover or perch , advanced flight controllers may adapt parameters online, potentially customizing controller parameters to special maneuvers such as transition to and from hover [4]. While special-purpose controllers have been successful at transition to/from hover, robust general-purpose aerobatic control systems for small UAS have been more elusive due to the unsteady aerodynamics encountered during operations at high angles of attack and due to the limited sensing capabilities typically available onboard small UAS compared to, for example, an advanced fighter aircraft. The proposed notion of aerodynamic sensing seeks to address small UAS control system limitations by providing strategies for vehicle feedback control based on onboard flow measurements, now made possible through emerging low-cost small-scale pressure sensors. Autopilots have traditionally relied on inertial sensor systems to detect disturbances and on linearized aerodynamic models to relate control surface deflections to resultant vehicle moments. This approach is well suited to forward flight conditions when flow conditions are attached but such controllers are not capable of predicting the effects of unsteady, non-linear aerodynamic related to high angle of attack flight. By incorporating direct measurements of the flow field surrounding a flight vehicle, a traditional inertial based autopilot can be enhanced by through constantly updated models of control surface effectiveness and predictions of imminent disturbances.

This paper describes the development of an aerodynamic feedback scheme for use on small UAS operating in unsteady flow conditions. In previous work, a pressure based aerodynamic instrumentation package was developed and implemented on a small fixed wing UAS alongside conventional instrumentation for autonomous flight [5]. It then documents the expansion of the aerodynamic sensing concept through system upgrades and new instrumentation. Flight test results from autonomous outdoor cruise-hover transitions flown by the first generation system are discussed, followed by open loop flight test data obtained through the upgraded aerodynamic data system.

\section{Background}

The aerodynamics of high angle of attack flight presents a number of challenges to autonomous UAS. Near stall, aircraft lift coefficients are non-linear with respect to parameters such as angle of attack and airspeed. Lateral handling issues such as asymmetric wing stall and wing rock also pose challenges at high angles of attack. Early research focused on mitigating manned aircraft handling issues near stall or enhancing fighter jet maneuverability [6,7]. This body of knowledge remains applicable to unmanned air vehicles, though recent focus has been on exploiting some of these capabilities for UAS.

Wickenheiser [8] considered large lift and drag coefficients to be useful for the ARES-C Martian Exploration Vehicle, and employed a lifting-line-based analysis of the longitudinal aerodynamics of an ARES reconfigurable exploration craft performing a form of fixed wing perching. Researchers have also worked on the closely related concept of flapping wing vehicle perching, and some recent progress by Paranjape et al [9] has demonstrated the ability of a tail-less morphing wing glider to perch autonomously by actuating its dihedral angle.

A range of small fixed wing UAS have been guided between cruise and hover in the last decade. Green and Oh developed indoor hobby aircraft that could autonomously transition from cruise to hover using inertial measurements and a linearized controller [10]. This impressive first autonomous fixed wing hovering work did not deal with the high angle of attack flight regime, using airframe properties such as low rotational inertia, high thrust/weight ratios and a control law that allowed it to "bully its way through stall". A similar approach in avoiding the aerodynamic problems at high angles of attack using similar flight vehicles by Frank et al[11] also achieved 
successful autonomous transitions to hover and docking in a VICON motion capture environment. More recently, Cory and Tedrake [12] used the VICON system to provide valuable insight into the non-linear aerodynamic effects encountered during a perching maneuver. This was a step towards exploiting the aerodynamic phenomena at stall instead of avoiding it. Using VICON position data, instantaneous aerodynamic coefficients could be estimated. It was noted that while individual trials showed time-varying coefficients due to unsteady aerodynamic effects, the overall trend of averaged data over many trials agreed with simple flat plate theory. This allowed the formulation of an aerodynamic coefficient estimator based on angle of attack and elevator deflection given still indoor air and the kinematic data from the VICON environment.

On a larger scale and outdoors, the wing rock characteristics of a medium sized Radio Control (RC) hobby aircraft flying at high angles of attack were studied by Lind and Johnson[13]. The aircraft was flown open loop by a $\mathrm{RC}$ pilot through multiple passes during which it experienced uncommanded wing rock. Johnson et al [3] developed an adaptive controller that enabled autonomous transitions to and from hover. The guidance law used during the transition was similar in formulation to those used in previous work - the commanded inertial pitch angle was set to vertical to achieve the transition. A slow ramp approach and a faster step-change transition were tested and it was noted that both resulted in a degree of altitude error during the transition. Johnson et al suggested that an airspeedbleed strategy was a potential solution. In their previous work [5], the authors proposed using pressure based aerodynamic sensing to support such a transition guidance phase. Processing pressure data over an instrumented wing chord, the autopilot was able to detect stall and could reliably bleed airspeed up to the point it was detected before switching control modes. Flight testing of this phase showed it was viable for a simple decoupled autopilot to stabilize the aircraft to the point of stall detection by the onboard pressure instrumentation.

The concept of pressure based estimation of the flow field above an airfoil has been a cornerstone of wind tunnel testing, but in stepping towards aerodynamics-based feedback, the most relevant efforts to our work have focused on enabling closed loop feedback in "onboard" active flow control schemes to alleviate flow separation or emulate control surfaces through the use of flow actuation. One successful example was implemented by Patel and Corke $[15,16]$ who considered the time domain response from a high bandwidth pressure sensor to predict incipient flow separation at the wing leading-edge and trigger the activation of a plasma flow actuator.

For small UAS applications, the authors have previously used a pressure based strategy to measure the unsteady aerodynamic forces over a set of flapping wings in a wind tunnel test environment with the intent of implementing control through flap stroke actuation [14]. Under attached flow conditions, Cox et al [17] used pressure based estimates of the lift curve above an airfoil as feedback for an automated a cruise flap. NASA has supported wind tunnel based implementation and testing of a distributed actuation and sensing array for use on a blended wing body UAS, using a series of pressure measurements to study the effectiveness of a morphing wing type control strategy. More information on these tests can be found in [18] and [19]. The AFOSR AVOCET project [20] aims to continuously tailor the pressure distribution and resulting forces and moments across the wing using advanced micro-tuft sensors and hybrid fluidic flow actuators.

The overall goals of the aerodynamic feedback concept developed in this work bridges the two efforts escribed above. The existing active flow control framework developed by Patel et al [ 15,16] is suited to alleviate retreating blade stall in rotorcraft and expand their performance envelope. With the AVOCET project, flow measurement and actuation across the wing surfaces are closely coupled to vehicle control and the system aims to achieve a careful tailoring of vorticity distributions in real time to also alleviate gusts and flow disturbances. While the intended purpose of the aerodynamic data presented in this work is to offer additional data for feedback control, our objectives are not to directly affect the flow structure but to provide improvements on how the conventional surfaces can be used. To this end, the authors have implemented a pressure based aerodynamic data system on a Funtana RC stunt plane and developed a preliminary transition guidance law that switches between discrete controller modes based on stall detection. Below, we present the aerodynamic instrumentation used for data collection and flight control experiments and recent upgrades to the aerodynamic sensing package are documented. Next, autonomous test results are presented from complete transition maneuvers using our first generation aerodynamic data system followed by open loop flight data taken with the improved aerodynamic sensing package.

\section{Aerodynamic Instrumentation}

A pressure based aerodynamic sensing system has been developed that augments the available vehicle state information available through inertial sensors during maneuvers that involve in unsteady aerodynamics. When operating in unsteady flow conditions such as past stall at high angles of attack, linearized aerodynamic models are less suitable to predicting the aerodynamic forces on a fixed wing vehicle. In these situations, the linearized 
relationship between control surface deflection and resultant aerodynamic forces is not directly applicable and external disturbances cannot be detected until the vehicle is affected by them. Extending the paradigm of feedback control, taking measurements around the vehicle during maneuvers outside of forward flight can provide additional and relevant flow information that will be otherwise difficult to predict. The aerodynamic sensing package is based on a set of pressure measurements taken across the aircraft through pressure ports and through multi-hole probes. . A schematic of the concept is shown in Figure 1 where components colored in red are currently operational. This section documents the ongoing development and flight testing of an aerodynamic sensing for fixed wing flight at high angles of attack.

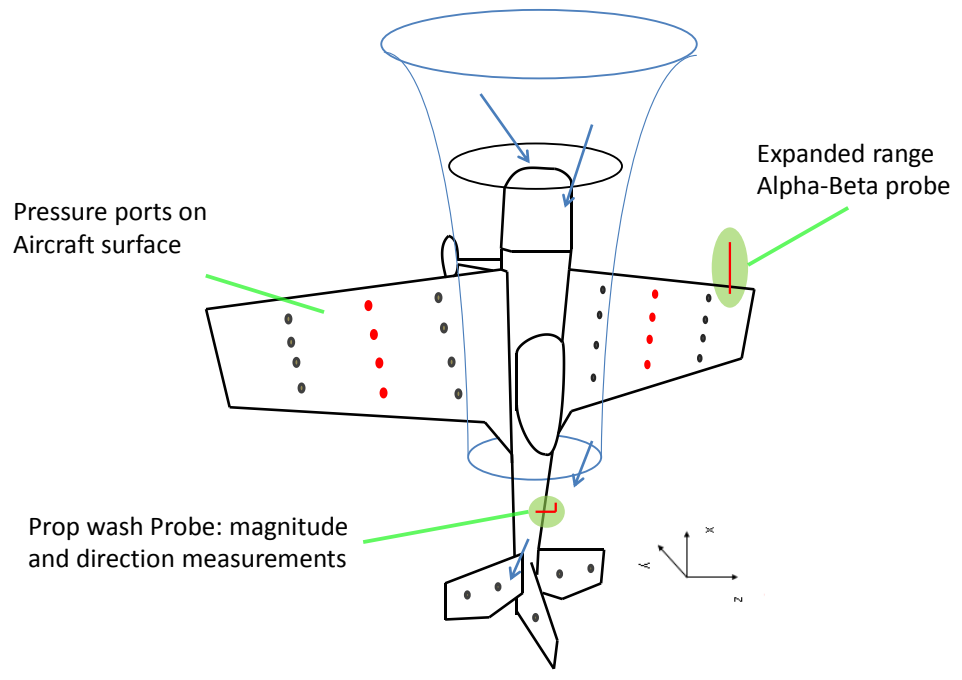

Figure 1: Pressure Based Aerodynamic Sensing Concept.

\section{A. Flight Vehicle}

The experimental test vehicle used is a fixed wing UAS with a first generation aerodynamic data system capable of performing autonomous, outdoor cruise-to-hover transition maneuvers. In previous work, the authors instrumented a commercially available Funtana X100 aerobatic RC aircraft with the avionics necessary for outdoor autonomous flight and a first generation aerodynamic sensing system. A custom designed and calibrated expanded range $\alpha-\beta$ probe and a single set of wing mounted pressure ports provided aerodynamic data such as high angle of attack measurements and basic stall detection. Two generations of the Funtana platform with upgrades in 2012 are shown in Figure 2 and additional details on the airframe and avionics are provided in our earlier work [5].
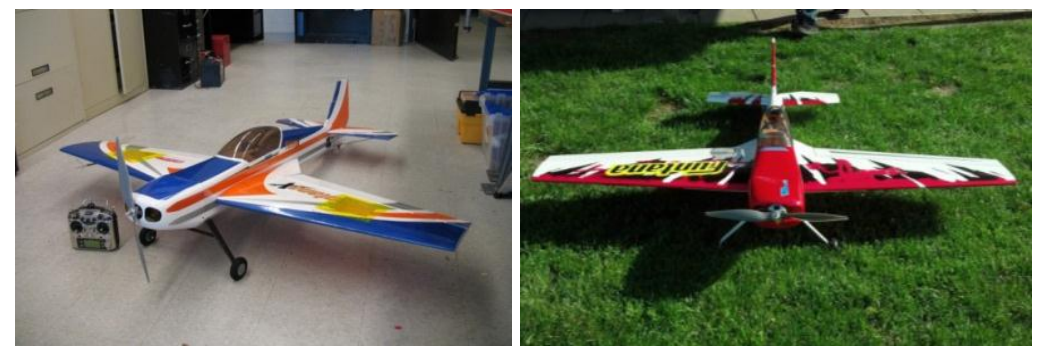

Figure 2: Funtana Test Aircraft - 2009 (left) and 2012 (right) versions

\section{B.Expanded wing port array}

The instrumented wing concept from[5] has been expanded in this work to offer better coverage and measurement accuracy in flight regimes where aerodynamic feedback can be most valuable. Using a different type of smaller pressure sensor allows for pressure sensors to be located near pressure ports, allowing improved response 
times and greater flexibility in locating pressure measurement points, such as within the ailerons. The pressure sensors were assembled and embedded along the pressure ports to avoid long pressure lines and long response times. The ports were fabricated from aluminum tubing and rubber hosing depending on where the port needed to be embedded. These are shown in Figure 3. The aircraft currently has one instrumented chord on either wing and more ports are being added as flight testing continues.
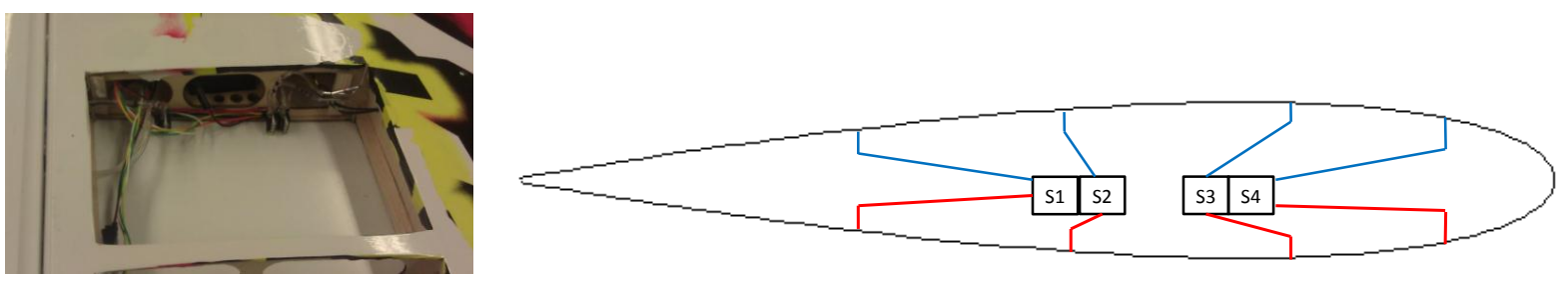

Figure 3: Wing Ports - Sensor Placement and Presure Line Routing Schematic

As with the previous system, the embedded pressure ports offer an autopilot the ability to estimate the lift being generated by each wing. With the new configuration however, the pressure sensors are connected to give differential pressure between ports located at the top and bottom wing surfaces instead of between individual ports and the leading edge. This allows a direct and straightforward estimate of lift that is more suited to flight at very low airspeeds. This avoids normalization difficulties associated with small dynamic pressure and allows a wing lift estimate to be made with fewer measurements, reducing the effects of sensor noise. The authors implemented a similar differential pressure sensing and integration concept with instrumented flapping wings and found it offered good estimates of unsteady aerodynamic lift [14]. Using differential pressure measurements, a coarse discretization and area integration scheme, lift estimates from pressure sensor measurements in unsteady flow conditions can be calculated. Adapted for the Funtana wings, the proposed discretization scheme is shown in Figure 4 where total lift can be estimated as in Equation (1). The mid-span ports on either wing have been embedded and port locations are being finalized based on ongoing flight test results.

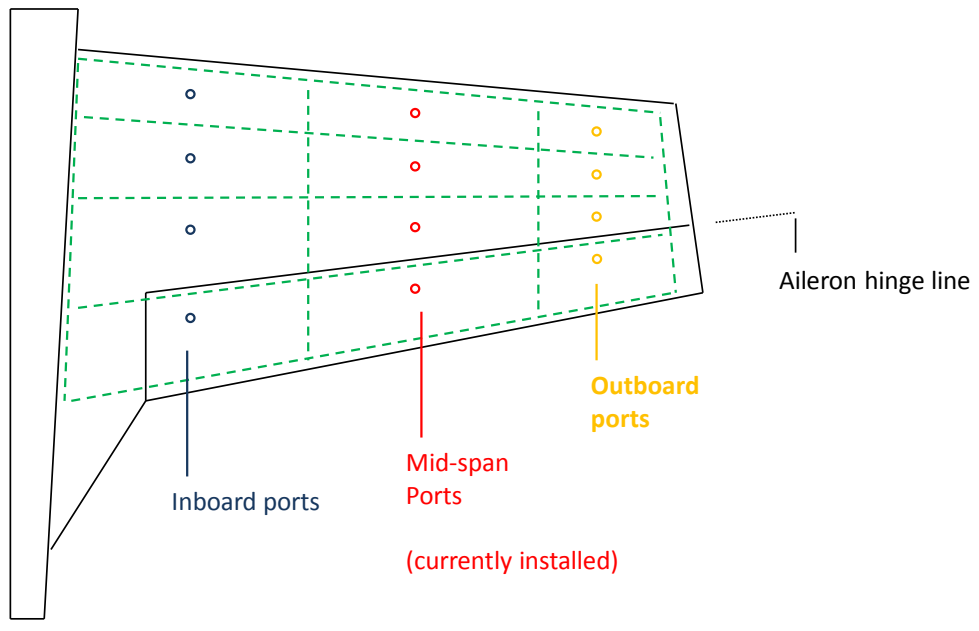

Figure 4: Proposed Wing Discretization for Pressure Based Lift Estimation

$$
\mathrm{L}=\sum_{i=1}^{\mathrm{n}} \mathrm{P}_{\mathrm{diff}-\mathrm{i}} \cdot S_{i}
$$

With the instrumentation expanded to both wings, the new pressure port network offers a variety of capabilities that were not possible when only one chord on one wing was instrumented. Being able to estimate lift generated by 
either wing at high angles of attack allows for the aerodynamic roll moments generated by the wings due to external disturbances or non-linear aerodynamics to be estimated.

\section{Updated stall detection scheme}

In the traditional case, an airfoil is pitched beyond a critical angle of attack will begin to generate a decreasing lift coefficient with increasing angle of attack. This departure from a mostly linear relationship causes a loss of lift and sudden changes in the aerodynamic forces generated by the aircraft. The new pressure measurement scheme has allowed the stall detection algorithm used in the first generation system to be further developed. With the differential pressure being monitored at more locations, the relative differences between pressure measurements from the leading edge and trailing edge can be compared with against each other, allowing a degree of stall prediction capability. The pressure port configuration at each wing chord is shown in Figure 5. The revised stall detection and prediction algorithm currently used in flight testing is presented in Equations (2) and (3) below. More detail on the development of the stall prediction algorithm is provided in the flight test results section.

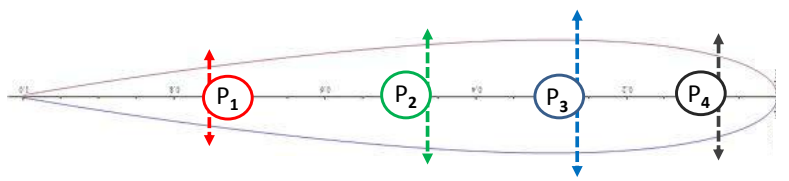

Figure 5: Chord-wise Pressure Port Locations and Differential Sensor Connections

$$
\begin{aligned}
& P_{\text {Profile }}=\left[\mathrm{P}_{1}, \mathrm{P}_{2}, \mathrm{P}_{3}, \mathrm{P}_{4}\right] \\
& \mathrm{P}_{\text {diffs }}=\sum_{i=1}^{n-1}\left|\mathrm{P}_{\mathrm{i}+1}-\mathrm{P}_{\mathrm{i}}\right| \\
\text { Wing Stall State }= & \begin{array}{lc}
\text { Full Stall } & \mathrm{P}_{\text {diffs }}<\mathrm{P}_{\text {diffs }_{\min }} \\
\text { No Stall } & \text { Otherwise }
\end{array}
\end{aligned}
$$

\section{D.Prop-wash probe}

While the single air data probe can provide expanded angle of attack and sideslip data to the autopilot, it was still not capable of providing the degree of flow-field information needed for high angle of attack flight. During first generation autonomous transitions, pitch oscillations due to excessive autopilot feedback gains were observed when switching between airspeed bleed and pitch-up phases. While the airspeed of the stalled aircraft was low, it was apparent that the control authority due to the incident prop-wash on the tail sections was substantial when the propeller was spinning at high rpm. This causes difficulties when attempting to use a linearized controller with gains scheduled according to airspeed. Operating at high angles of attack where propeller thrust can be responsible for most or all the lift being generated, a single air data probe and a single point of measurement was not capable of providing enough information about the flow field surrounding the aircraft.

To provide additional measurement data for the controller, a new fuselage-mounted multi-hole probe was designed and built using aluminum tubing and mounted in front of the tail section. The probe provides flow angle and speed measurements by comparing the pressure measured across its prongs and is configured to use three differential pressure sensors. The geometry of the probe and a picture of it mounted on the fuselage is shown in Figure 6.

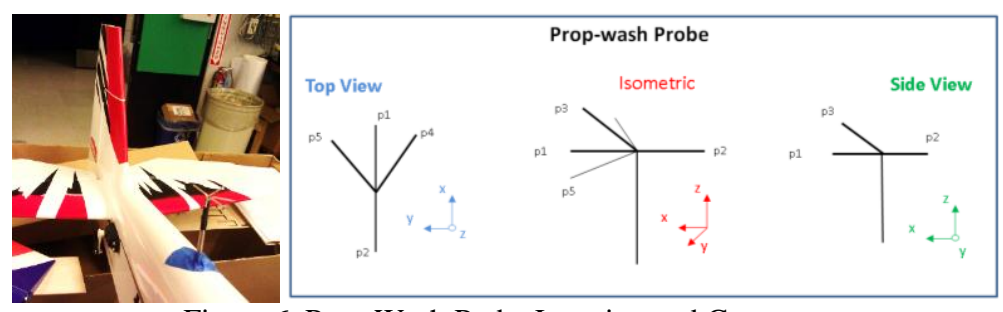

Figure 6: Prop-Wash Probe Location and Geometry 
The current prop-wash sensing scheme is intended to provide only rough estimates of the flow field, and the probe is designed to be easily replaced with a simplified calibration. The probe is calibrated for a smaller range of inflow angles than the wing-mounted $\alpha-\beta$ probe since the anticipated range of prop wash flow angles is relatively limited, and the coupling of yaw and pitch measurements is ignored. Calibration was performed in the University of Michigan 2x2 instructional wind tunnel. The probe was mounted on a rotational stage and data was taken at flow angles between -60 and 60 degrees at seven different $V_{\text {air }}$ between $3 \mathrm{~m} / \mathrm{s}-21 \mathrm{~m} / \mathrm{s}$. The first and second prongs were aligned with the wind tunnel flow by sighting down lengths of thread. Due to the use of forward/backward prongs, the reference dynamic pressure quantity changes slightly with inflow angle. This causes the calibration curves for probe- $\alpha$ and probe- $\beta$ to be linear only for small flow angles. It was found that a $7^{\text {th }}$ degree polynomial provided an adequate approximation. The data reduction and calibration scheme is described by Equations (4) through (6). The resulting calibration curves for prop-wash inflow angle are shown in Figure 7 for angles between -45 and 45 degrees.

$$
\begin{aligned}
& \Delta \mathrm{q}=\mathrm{p}_{1}-\mathrm{p}_{2} \quad \Delta \alpha=\mathrm{p}_{3}-\mathrm{p}_{1} \quad \Delta \beta=\mathrm{p}_{4}-\mathrm{p}_{5} \\
& \alpha=\mathrm{K}_{\alpha 7}\left(\frac{\Delta \alpha}{\Delta \mathrm{q}}\right)^{7}+\ldots \mathrm{K}_{\alpha 3}\left(\frac{\Delta \alpha}{\Delta \mathrm{q}}\right)^{3}+\mathrm{K}_{\alpha 2}\left(\frac{\Delta \alpha}{\Delta \mathrm{q}}\right)^{2}+\mathrm{K}_{\alpha 1} \frac{\Delta \alpha}{\Delta \mathrm{q}}+\mathrm{K}_{\alpha 0} \\
& \beta=\mathrm{K}_{\beta 7}\left(\frac{\Delta \beta}{\Delta \mathrm{q}}\right)^{7}+\cdots \mathrm{K}_{\beta 3}\left(\frac{\Delta \beta}{\Delta \mathrm{q}}\right)^{3}+\mathrm{K}_{\beta 2}\left(\frac{\Delta \beta}{\Delta \mathrm{q}}\right)^{2}+\mathrm{K}_{\beta 1} \frac{\Delta \beta}{\Delta \mathrm{q}}+\mathrm{K}_{\beta 0}
\end{aligned}
$$
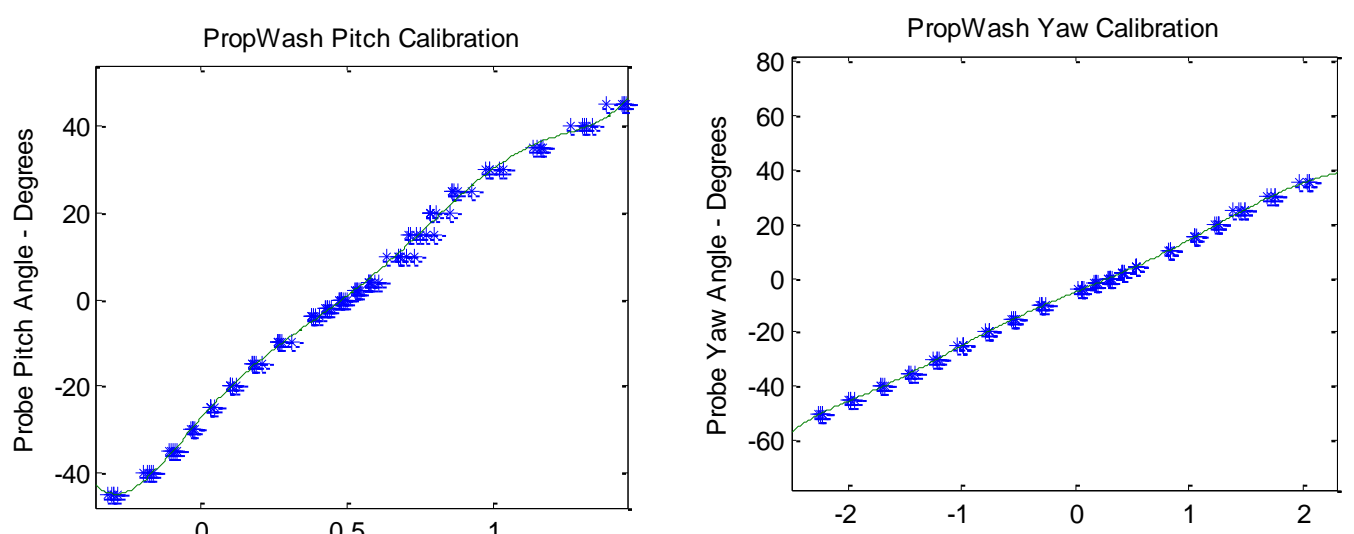

Top/Total Prong Readings by Dynamic Prong Measurements Beta Prong Readings by Dynamic Prong Measurements

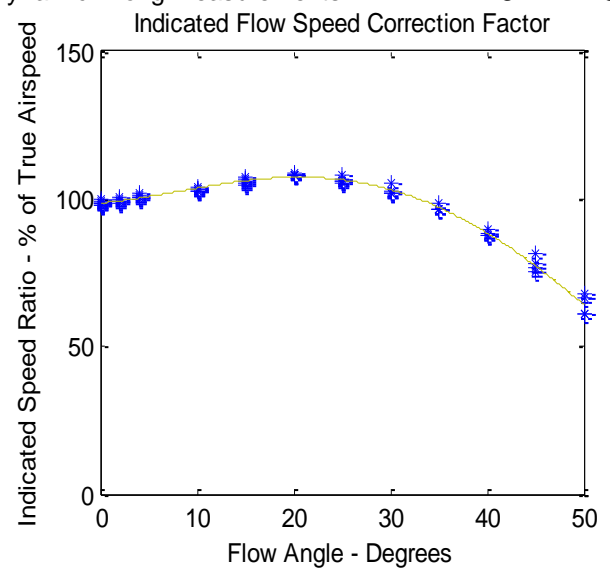

Figure 7: Prop-Wash Probe Flow Angle Calibration Curves 


\section{E.Revised $\alpha-\beta$ Probe Calibration Scheme}

The multiple curve calibration scheme described in our previous work [5] was refined and extended in range to enable flow angles up to 80 degrees. A large angle range calibration technique was previously implemented to accommodate a larger range of flow angles, allowing angles of attack up to 75 degrees to be effectively measured. This was made possible by switching reference pressure for measurement normalization at larger angles of attack and using three separate calibration curves. For small angles, a curve that relates the pressure difference between the $\propto$ prongs normalized by dynamic pressure is valid. For larger inflow angles, two curves that relate the reverse normalization variable to angle of attack are valid for positive and negative flow angles.

In the previous system, a filter that tracked flow angle history was used to choose between calibration curves in real time. With the new sensor configuration, the direction of the probe can be obtained by the direction of the pressure difference between prongs. With the new algorithm, the pitot probe measurement of dynamic pressure is first normalized by the $\propto$ prong pressure difference, giving a reverse-normalization variable. By taking into account the magnitude and direction of the reverse-normalization variable, the appropriate calibration curve can be determined uniquely. The map shown in Figure 8 presents ranges of reverse-norm values and the appropriate calibration curve that was chosen.

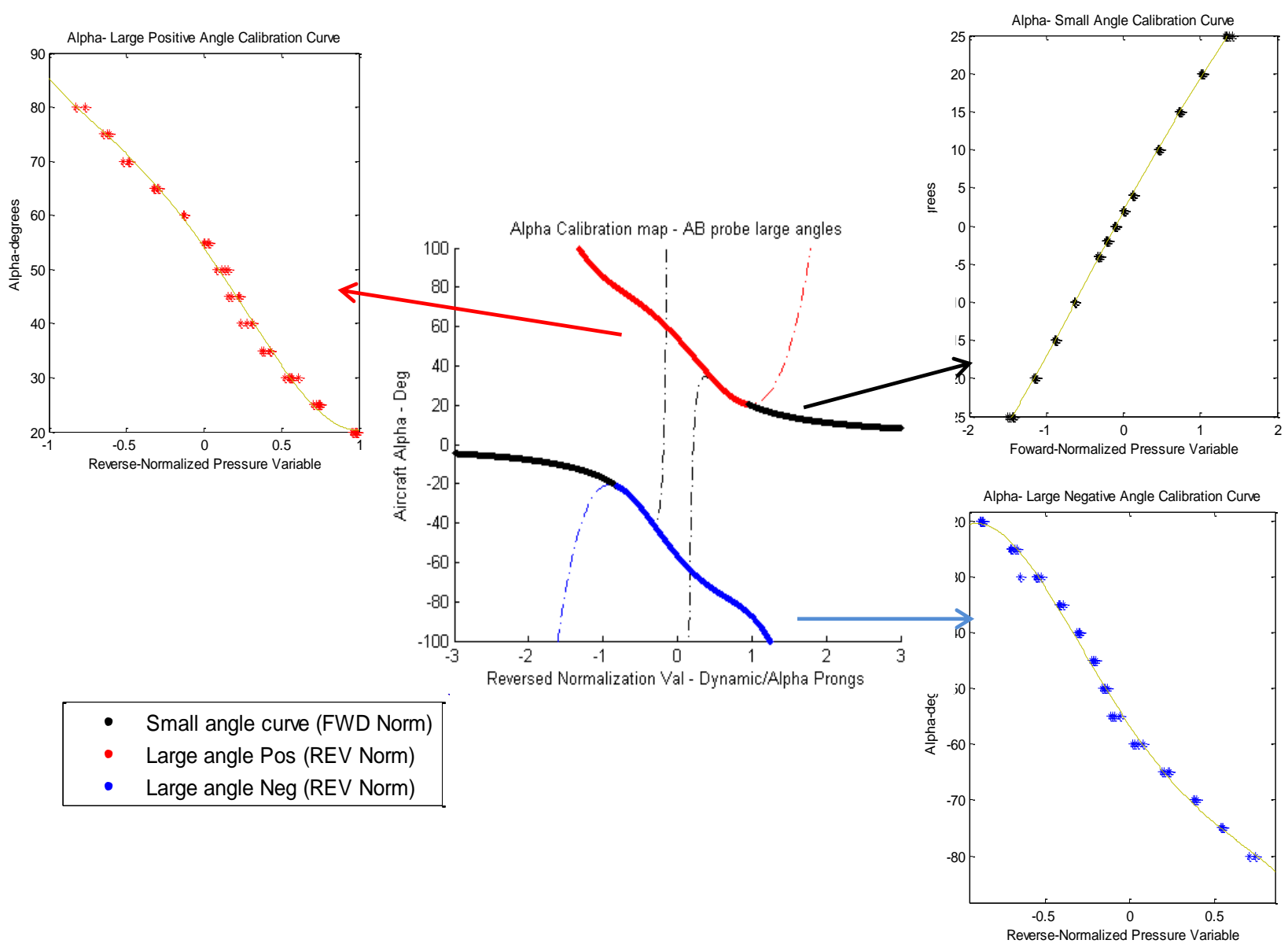

Figure 8: Sample Expanded Range Curve Selection Map for $\propto$ 


\section{Flight Test Results}

\section{A. First Generation Instrumentation: Autonomous Transitions}

The first generation aerodynamic sensing scheme was integrated into a cruise to hover transition autopilot and flight tested. Outdoor autonomous transitions were previously performed outdoors by Johnson et al [4] using an adaptive neural network controller to successfully control the aircraft solely on inertial measurements in both orientations. The autopilot used by Johnson et al to transition the aircraft through high angle of attack flight and stall was open loop with respect to the aerodynamics. Only inertial pitch angle references were used during transitions and no measurements of the flow conditions were taken. Johnson et al found that linearly changing reference pitch and airspeed resulted in significant altitude change during a transition. They noted that future work will require an 'airspeed bleed' scheme.

It was thought that with additional flow measurements, a simple autopilot could be implemented during such a phase when the aerodynamic forces were unsteady. A key feature of the airspeed bleeding process is encountering stall. A transition guidance law was proposed that inserts an additional "Trans LP" phase where airspeed is bled until the aircraft has flown into a stall before switching to "Trans HP" which is a phase comparable to the transition strategy previously implemented. The transition guidance used for first generation autonomy is shown in Figure 9.

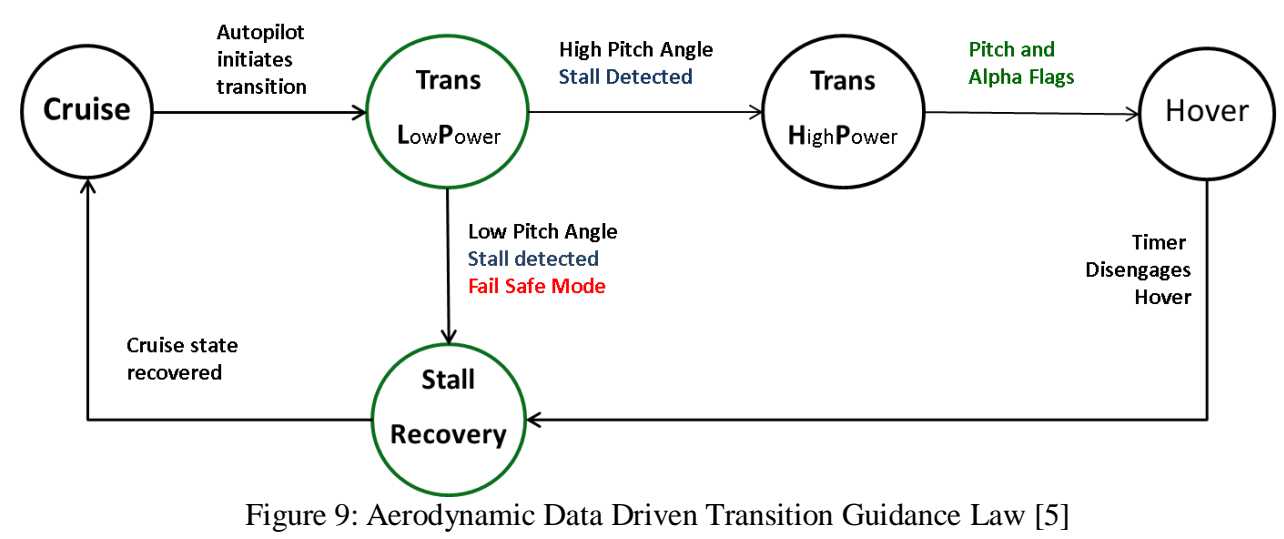

An additional stall recovery phase was added in case the autopilot failed to detect stall until the nose of the aircraft had already pitched downwards from level flight. In this phase, the autopilot aborts the transition and returns to a cruise state.

The aerodynamic-sensing enabled guidance scheme was combined with a decoupled lateral/longitudinal autopilot for tests. Stall detection data and expanded angle of attack data is used to trigger mode changes. Airspeed data is used to schedule gains. During a typical test flight, the aircraft is first taxied and taken off remotely by a RC pilot. Once the aircraft is lined up for a straight flight pass, the autopilot is given control of the aircraft. The autopilot begins in the cruise mode where the wings are leveled using the inertial frame. The pitch reference is set based on an altitude hold loop which uses the initial GPS altitude as a reference. The heading at initialization is also used as the heading reference for cruise and subsequent modes until the autopilot is deactivated. Once the aircrafts has reached a stable cruise and the rotation rates decrease below a set threshold, the autopilot self-initiates the Trans LP airspeed bleed phase. The motor is powered off and the autopilot attempts to maintain a the cruise inertial pitch angle at the time of mode switch while the airspeed decreases. This causes the autopilot to increase elevator deflection to maintain a level pitch angle until stall is detected by the onboard computer using the stall detector describe. Unless the aircraft has entered a nose-down dive with an inertial pitch angle of below -10degrees, Trans HP is initiated. This phase executes the remainder of the transition in a way similar to that used by other researchers. The throttle is increased to maximum and the reference inertial pitch angle is set to vertical. The autopilot recognizes a hover state once a near vertical pitch angle and a sufficiently high angle of attack is measured. For the first generation, there is no dedicated hover controller and the aircraft recovers after a 2 second delay. In the recovery phase, the autopilot points the nose downward while increasing throttle until stall is no longer detected and switches to a cruise state. Data from a single transition is shown in Figure 10. Angle of attack is compared against inertial pitch angle and wing pressure port data is presented along with stall detection results. The GPS track and airspeed are also presented. 

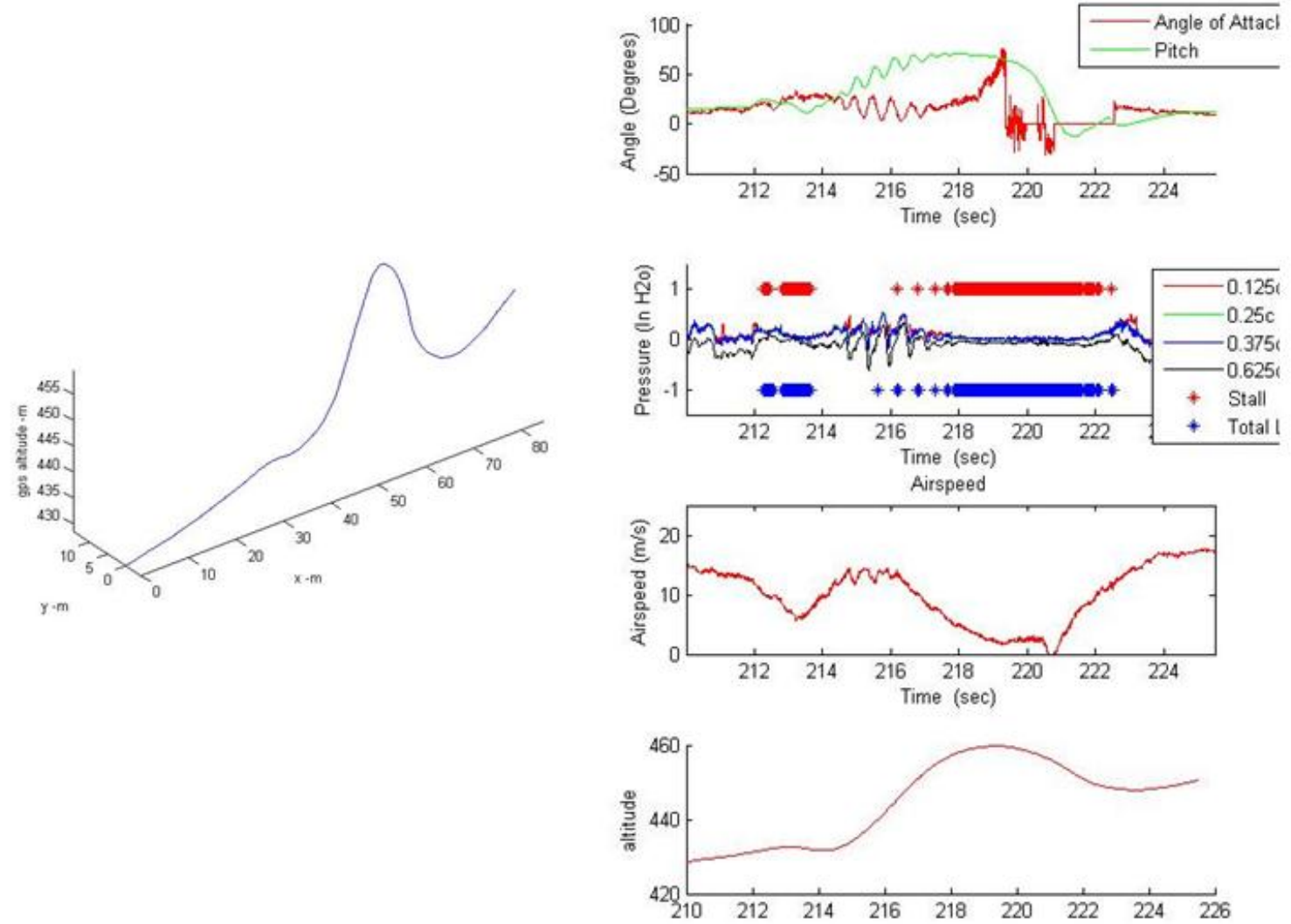

Figure 10: Sample Autonomous Cruise to Hover Transition Data

Flight testing shows that the controller provided reliable stall detection and mode switch triggering. It performed repeatable and consistent transition maneuvers consecutively despite the unsteady aerodynamics involved. From the data the autopilot consistently recognized stall and switched modes to Trans HP. However, the high power phase encountered problems with large oscillations in pitch resulting in an extended vertical climb and excursions in altitude. This trend result was repeated consistently throughout different flights. The plots in Figure 11 show this trend occurring over a sequence of four consecutive transitions. Average altitude increase across a transition maneuver was approximately $25 \mathrm{~m}$ after factoring in a constant climb rate during cruise. The large oscillations in pitch during the high power transition phase prevented smooth rotation into a hover orientation at low airspeed. Despite this, the ability to consistently bleed airspeed and fly into a stall meant vertical excursions were comparable to those achieved with more advanced controllers suggesting that the strategy could be tuned to afford improved vertical accuracy during cruise-to-hover transitions with simple control strategies.

While the airspeed bleed phase appears to function well, the Trans HP phase can benefit from a number of changes. With no feedback on motor RPM or thrust, the throttle ramp was set to maximum during Trans HP, causing an excessive thrust for the weight of the aircraft. It can be noted in the data that when the aircraft is pitched upwards, the airspeed actually approaches that of the entry cruise airspeed and the wings are seen to have recovered from stall. While the performance of Trans HP might be improved through tuning the open loop parameters in this application, it is believed that implementing simple feedback control strategies based on additional sensing can offer the most benefits for this type of maneuver. 

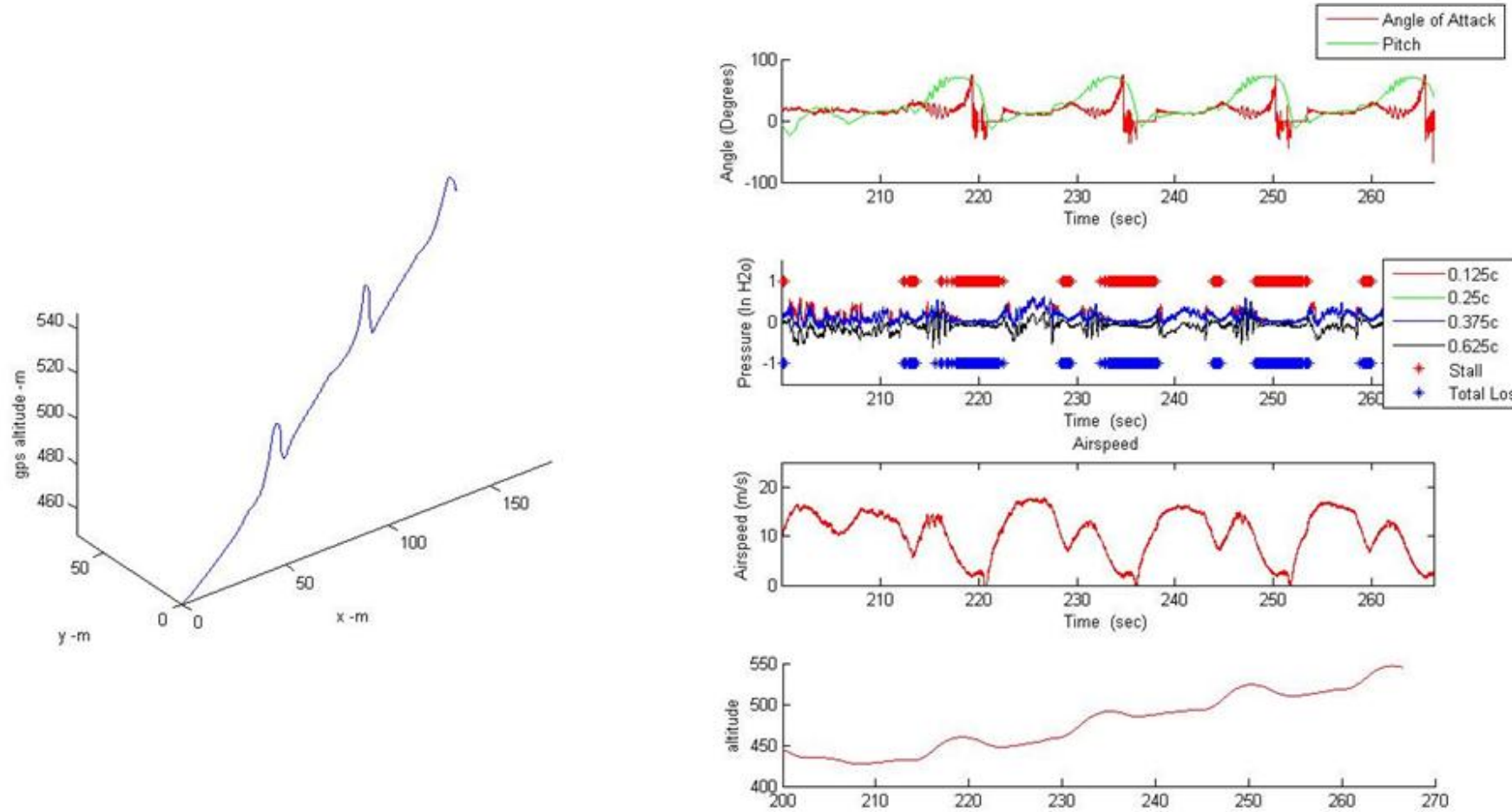

Figure 11: Autonomous Transiton Maneuver History - Four Consecutive Transitions

In the work described in this section, which was completed with flight testing at Camp Grayling MI in late 2009, it was determined that vehicle airspeed measurements alone were not capable of describing the flow field around the vehicle due to the significant prop wash over the tail surfaces and the dependence on propeller thrust for lift with no means of measuring it. This resulted in inaccurate gain scheduling for the elevator and an essentially open loop transition controller that was given control of a 2000watt propulsion system. This hypothesis and other lessons learnt from the autonomous flight tests guided the further development of the second generation sensing scheme, results from which are presented below.

\section{B. Remotely Piloted Flight Test Data - Second Generation Aerodynamic Sensing System}

The expanded aerodynamic data system was flight tested through a series of remotely piloted aerobatic maneuvers. In addition to cruise-hover transitions, high angle of attack passes with wing rock and snap rolls were executed to assess the new instrumentation during high angle of attack maneuvers. The wings-level transitions and forward-stalls allow data from the prop-wash probe to be evaluated. Asymmetric stall conditions present in rock and roll maneuvers might be better characterized by examining both left and right wing pressure datasets. The higher performance maneuvers involved substantial aircraft rotations in addition to largely separated flow conditions. Where applicable, data from the aerodynamic instrumentation is presented along with inertial measurements taken by an off-the-shelf Microbotics MIDG-II inertial navigation system (INS).

\section{i. Stall Prediction Data}

The presented stall detection system was used in a mode switching capacity (Figure 9) when the aircraft entered a stall condition for transition-to-hover. At stall, the aerodynamic lift generated by the wings begins to decrease with increasing angles of attack due to the domination of separated flow across the wing surfaces. As described earlier, the wing is considered to have entered a 'full stall' when the pressure distribution has flat lined along the instrumented chord line. This was a straightforward process since in a full stall the pressure distribution is simply a flat line which needs little work to detect. However, notification can only be reliably given after the aircraft has transitioned between an attached flow condition and fully separated flow condition with no advance warning of the 
significant changes in handling characteristics. Developing the concept further, a methodology for indicating the onset of flow separation across the top of the wing could benefit an onboard controller by providing advance indication of imminent changes in flight condition.

To develop a stall prediction algorithm based on our instrumentation scheme, the pressure port data leading up all recorded stalls flown by the aircraft were isolated and compiled. A number of pressure measurement based variables were formulated based on what is expected from flow separation, and their evolution is tracked during the period before a stall was detected. A sample pressure distribution plot that is characteristic of attached flow and the corresponding pressure measurements is shown in Figure 12. These variables are calculated using Equations ( 7) and (8).

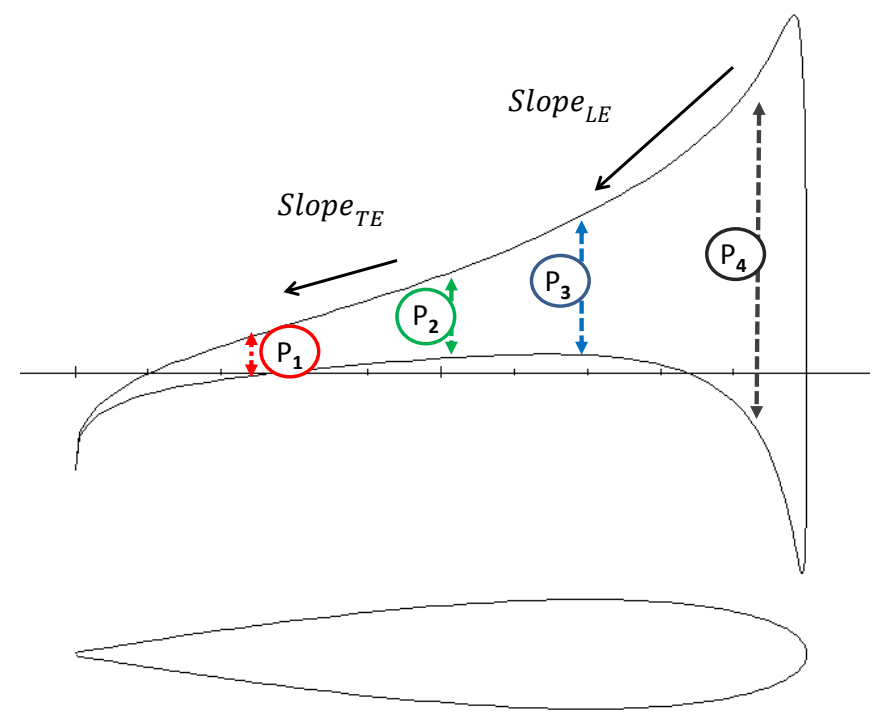

Figure 12: Pressure Measurement Overview and Stall Detection Variables

$$
\begin{gathered}
P_{\text {Profile }}=\left[\mathrm{P}_{1}, \mathrm{P}_{2}, \mathrm{P}_{3}, \mathrm{P}_{4}\right] \\
P_{\text {band }}=\max \left(P_{\text {Profile }}\right)-\min \left(P_{\text {Profile }}\right) \\
\text { Slope }_{L E}=\mathrm{P}_{4}-\mathrm{P}_{3} \quad \text { Slope }_{T E}=\mathrm{P}_{2}-\mathrm{P}_{1}
\end{gathered}
$$

It was found that the departure from attached flow conditions can indeed be reliably detected by monitoring the Slope $_{L E}$ variable, which is the difference between the first two measurement points near the leading edge. When the value of this variable changes sign or approaches zero, it is analogous to a loss of the suction peak characteristic of attached flow. This signals that stall conditions are imminent. On the Funtana platform, this can happen at low airspeeds when slowly decelerating, or a high speeds during aggressive maneuvering. In either case, stall detection is preceded by such a sign change of the Slope $e_{L E}$ variable. Approximately 40 cruise-stall maneuvers were recorded over a series of three flights. Due to space constraints, only the first four detected stalls are shown but the behavior

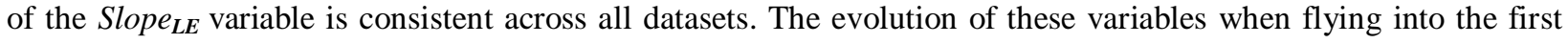
four stalls is shown in Figure 13 where each plot depicts a different remotely piloted stall from different airspeeds. 

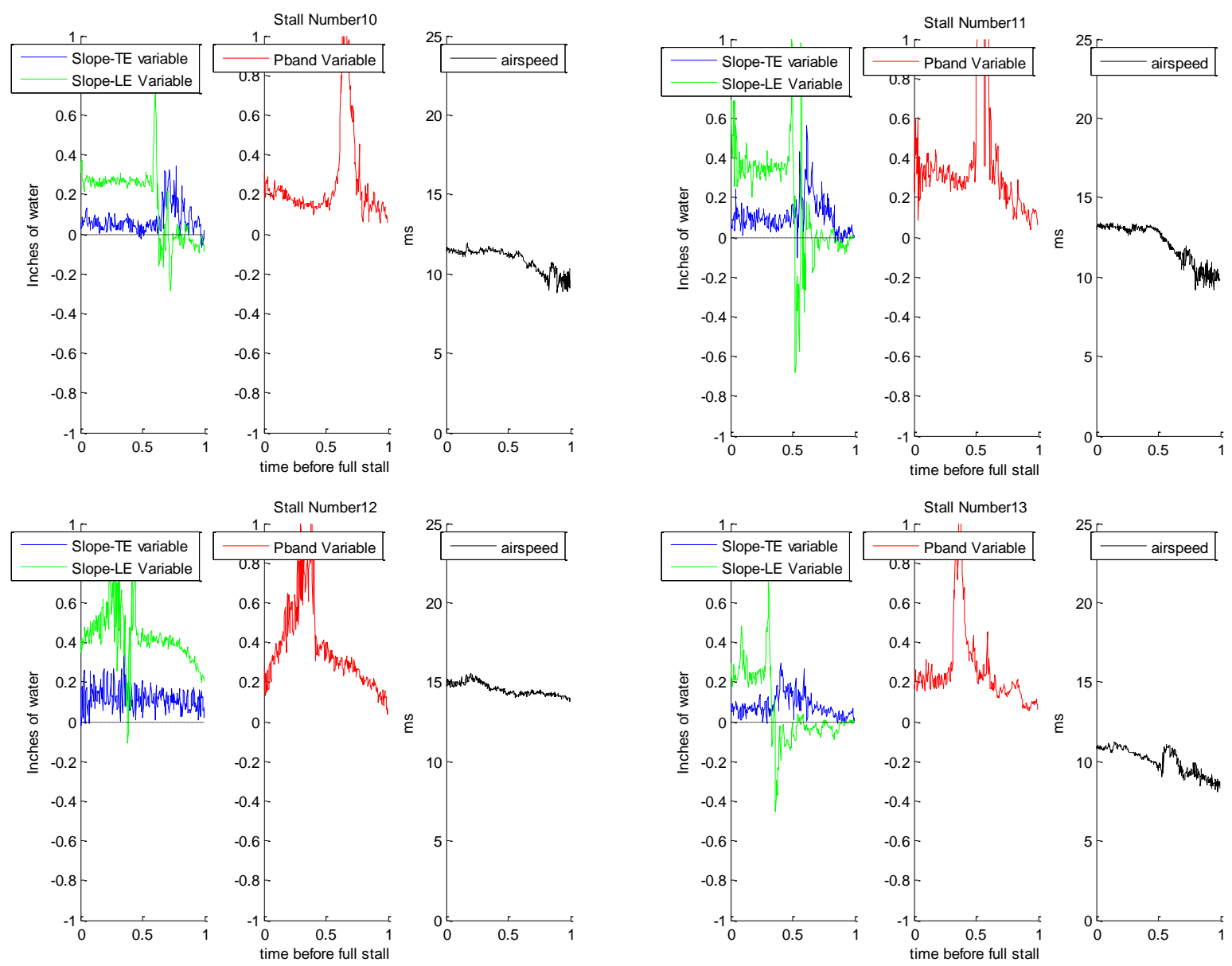

Figure 13: Stall Detection Variable History - Sample Set of Stall Maneuvers

It can be seen that before a stall is detected, a transition and sign change in Slope $_{L E}$ can be observed. This change is accompanied by an increase in the Slope $e_{T E}$ variable. It is expected that this is an indication of the transient shift in the pressure distribution due to vorticity being shed from the leading edge. The described trend is observed in reverse when the aircraft is cruising inverted and enters an inverted stall, as depicted in Figure 14. Work is ongoing to non-dimensionalize the maneuvers through establishing a convective time based on parameters such as airspeed and wing chord so data leading up to all recorded stalls can be plotted on the same axis to form a "Characteristic Funtana Stall" as measured by the pressure based system. If successful, this could provide the autopilot an alternative means of stall detection that does not require tracking the turbulence parameters in the flow and their histories [16]. 


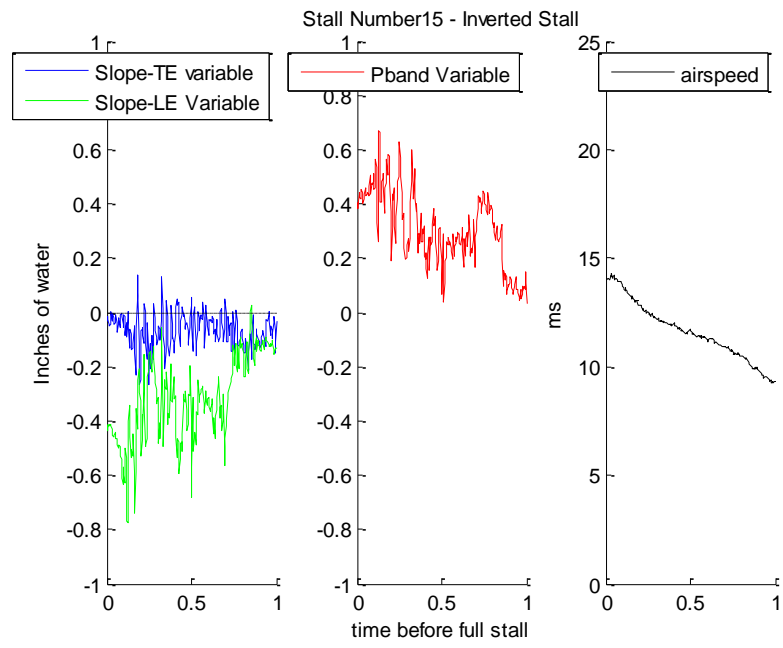

Figure 14: Stall Detection Variable History - Inverted Flight to Inverted Stall

\section{ii. Differential Lift Measurements - Onset of Uncommanded Wing Rock}

The left/right wing ports allow an initial test of flow measurement-based predictions of aerodynamic forces and resulting vehicle motion. Instances of un-commanded wing rock with no aileron input or throttle were initiated during which the differential lift measurements between left and right wings were compared against the roll rate histories measured by the IMU. During these maneuvers, the aircraft was flown at high angle of attack near 45 degrees with throttle held constant and aileron held at its neutral trim condition This makes the differential lift generated by left vs. right wing the primary source of aircraft roll moment. The onset of wing rock is shown in the Figure 15 and Figure 16 plots, where estimated aerodynamic moments are compared against measured roll rates from the IMU. It can be noted that in these situations, the lift-differential histories precede reported aircraft roll rates. This suggests that aerodynamic data can provide an advance indication of disturbances due to aerodynamic forces before inertial instrumentation can.

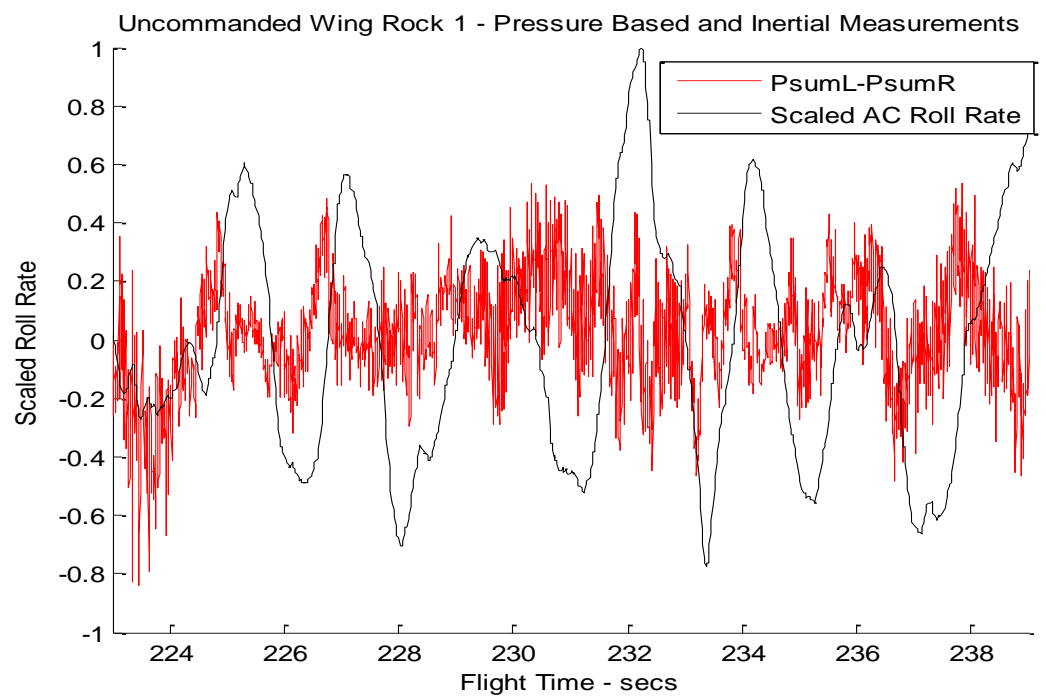

Figure 15: Uncommanded Wing Rock Onset - Maneuver 1 


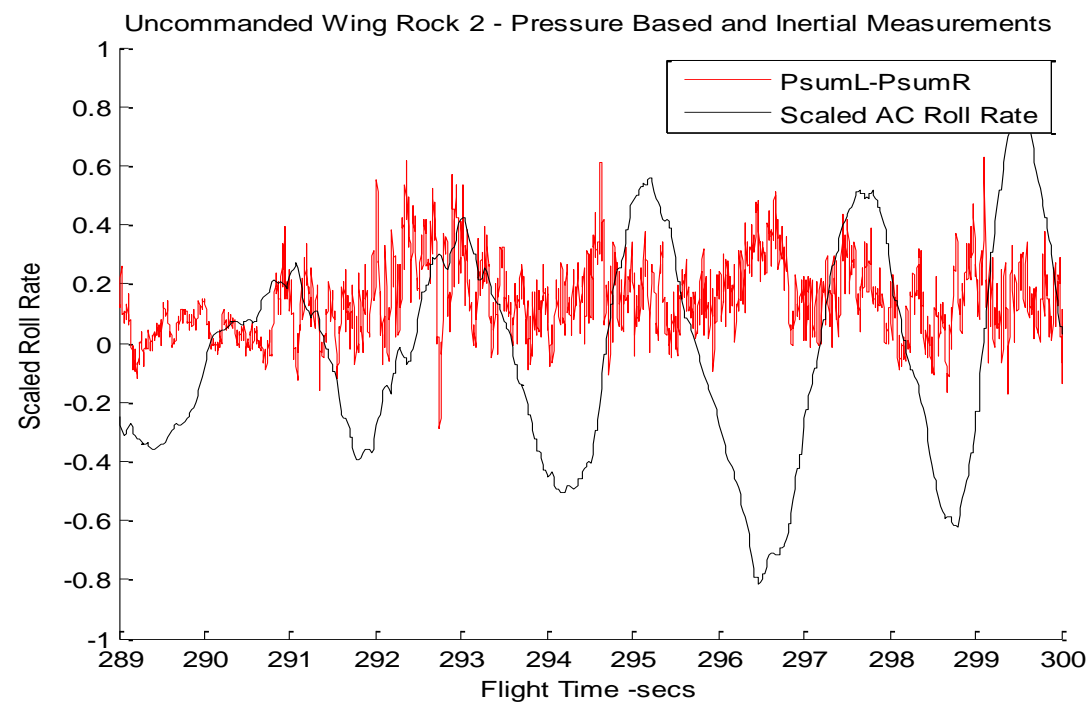

Figure 16: Uncommanded Wing Rock Onset - Maneuver 2

A preliminary characterization of this phase difference is summarized in Table 1. The periods of the aircraft roll rate signal over steady wing rock cycles were manually tabulated and compared against the differential aerodynamic lift signal for phase. This was done for data from two different flights The 12 periods from flight A were consecutive, but the 15 periods from flight B were from two different passes. For both flights, the average airspeed during the maneuver was $7 \mathrm{~ms}$ at an average angle of attack of approximately 45 degrees. While preliminary, the data indicates a degree of repeatability that warrants further testing.

\begin{tabular}{|c|c|c|}
\hline Flight A - 12 periods & & \\
\hline Average $\propto 45 \mathrm{deg}$ & Average & Stdev \\
\hline Average period (s) & 2.14 & 0.19 \\
\hline Average Aero-Advance (s) & 0.53 & 0.08 \\
\hline$\%$ phase advance & 24 & 0.03 \\
\hline
\end{tabular}

FlightB 9+6 periods

\begin{tabular}{|l|r|r|}
\cline { 2 - 3 } \multicolumn{1}{l|}{ Average $\propto 45 \mathrm{deg}$} & \multicolumn{1}{c|}{ Average } & \multicolumn{1}{c|}{ Stdev } \\
\hline Average period $(\mathrm{s})$ & 2.24 & 0.14 \\
\hline Average Aero-Advance $(\mathrm{s})$ & 0.48 & 0.15 \\
\hline \% phase advance & 21 & 0.06 \\
\hline
\end{tabular}

Table 1 : Average Phase Information from Two Flights

Aerodynamic-based predictions of aircraft roll rates can be helpful in controlling phenomena such as wing rock. Wing rock has been studied extensively and has been likened to a limit cycle oscillation driven by a form of aerodynamic hysteresis [21]. In a wind tunnel implementation of a fuzzy logic delta-wing stabilizing controller based on roll-rate feedback, Anavatti et al [22] found that actuation lag could cause a controller to be unsuccessful in reducing wing rock amplitude, requiring a manual phase shift to allow the actuation to occur at the appropriate time during the next cycle. Measuring the aerodynamic roll moments might afford additional lead time for future 
autopilots encountering wing rock. The aerodynamic forcing can potentially be used to tune a simple spring-massdamper to model the aircraft in the roll axis. While promising, more flight testing must be done to refine these results and to define flight parameters during which the use of differential lift measurements is useful to a controller. Data filtering can also be used to smooth noise in the raw aerodynamic measurements presented in Figures 15-16 to minimize the possibility of defining wing rock from transient noise in the data.

\section{iii. Prop-wash Probe: Cruise to Hover}

At high angles of attack when weight is primarily balanced by propeller thrust rather than lift, the difference in flow conditions between the wings and the tail section is most dramatic. The plots in Figure 17 show the ability of our prop wash probe to track the evolution of the prop-wash on the tail section during a remotely piloted transition and recovery maneuver. The flow field behind the prop is turbulent with intense velocity fluctuations, and a simple 3 point moving average filter was used for prop wash flow angle measurements. From the data, it can be seen that the longitudinal prop-wash velocity component tracks the indicated airspeed when entering the maneuver. Half way through the transition at an airspeed of approximately $10 \mathrm{~m} / \mathrm{s}$, the two flow velocities begin to differ dramatically. The previous generation autopilot that largely scheduled gains based on indicated airspeed during the deceleration phase was not able to account for this effect. During the transition and high angle of attack (>50degrees) flight, it was also noticed that the flow angles measured were large but within the limits of the calibration range.

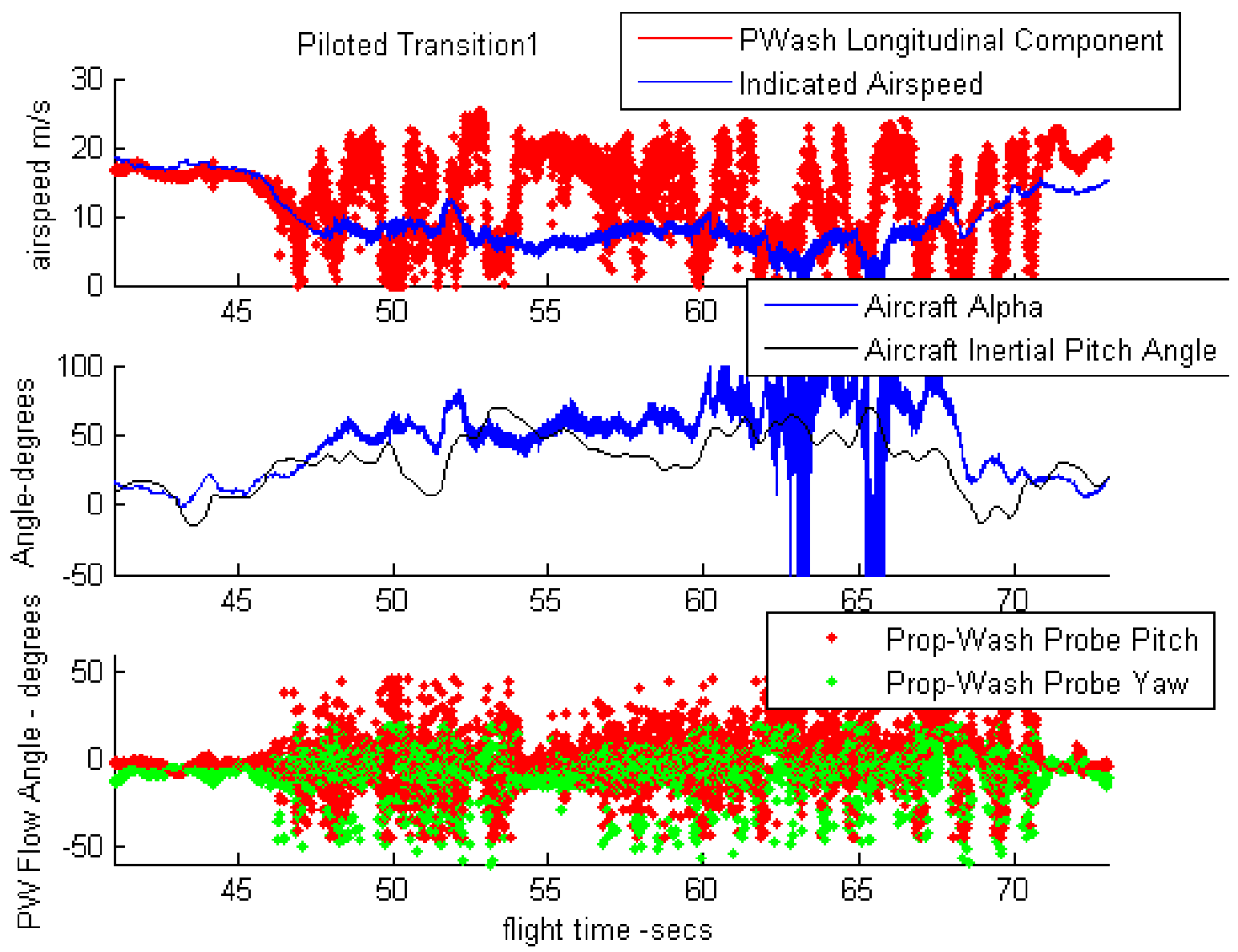

Figure 17: Prop-Wash Measurment during Piloted Transition at High $\propto$

It is hypothesized that accounting for the local flow incident on the tail surfaces will enable the transition autopilot described in an earlier section to improve its performance during the high power transition phase. Subject to the intense velocity fluctuations in the prop-wash, the relationship between control surface deflection and 
resulting pitch moment can be more accurately linearized about the incident flow even though the aircraft itself is flying through stall. The use prop-wash estimates over the tail to apply more appropriately scaled linear control gains for the tail surfaces is being investigated in ongoing work.

\section{Conclusions and Ongoing Work}

This paper has presented an aerodynamic feedback scheme for use on small UAS operating in unsteady flow conditions. A first-generation aerodynamic sensing scheme has demonstrated its ability to support a simple linear controller to detect stall during autonomous transition-to-hover maneuvers. Upgrades to the sensing package, including new embedded flow sensors and a prop wash probe, have been made to extend its capabilities. The new instrumentation functions as expected in flight. By combining simple aerodynamic principles, readily available materials and pressure transducers systems, a sensing and flight data scheme can be implemented that offer possibilities for small UAS flight control based on aerodynamic feedback. The control possibilities represented by the current package are currently being investigated. Remotely piloted and autonomous flight testing is expected to continue, with the short-term goal of using the new aerodynamic data to improve accuracy and robustness in a suite of transition and hover flight control laws.

\section{Acknowledgements}

This work has been supported in part by the Boeing Company. We also thank Mr. Jerry Lin for his help in the lab and at the flying field.

\section{References}

1 Y. Kang, B. Park, C. Yoo and S. Koo, " Control Law Modification According to Flight Test of Small Tilt Rotor UAV", Proc. AIAA Guidance, Navigation, and Control Conference, 18-21 August 2008, Honolulu Hawaii . (AIAA 2008-6334)

2 Festo (2011, Mar,) Festo. [Online]. http://www.festo.com/cms/en_corp/11369.htm

3 E. Johnson and S. Kannan, “ Adaptive Flight Control for an Autonomous Unmanned Helicopter”, Proc. AIAA Guidance, Navigation, and Control Conference, 5-8 August 2002, Monterey California . (AIAA 2002-4439)

4 Johnson, E., Wu, Neidhoefer, J., Kanna and Turbe. "Flight-Test Results of Autonomous Airplane Transitions Between Steady-Level and Hovering Flight," AIAA Journal of Guidance, Control, and Dynamics, Vol. 31, No. 2, March-April 2008.

5 D. Yeo, J. Henderson, and E Atkins, "An Aerodynamic Data System for Small Hovering Fixed-Wing UAS," Proc. AIAA Guidance, Navigation, and Control Conference, 10-13 August 2009, Chicago Illinois. (AIAA 20095756)

6 J. Chambers and S. Grafton, “Aerodynamic Characteristics of Airplanes at High Angles of Attack". NASA Technical Memorandum 74097, Langley Research Center, Hampton Virginia, 1977

7 J. Bauer, R. Clarke, J. Burken, "Flight Test of the X-29A at High Angle of Attack: Flight Dynamics and Control" NASA Technical Paper 3537, Dryden Flight Research Center, Edwards, California 1995

8 A. Wickenheiser, and E. Garcia, "Longitudinal Dynamics of a Perching Aircraft". Journal of Aircraft, Vol. 43, No.5, pp. 13866-1392.

9 A. Paranjape, S. Chung, H. Hilton and A. Chakravathy. "Dynamics and Peformanvce of Tailess Micro Aerial Vehicle with Flexible Articulated Wings". AIAA Journal of Aircraft, Vol. 50 No.5 May 2012 and Proc.AIAA Atmospheric Flight Mechanics Conference, Toronto, Ontario 2-5 August 2010. 
W. Green and P. Oh, "A MAV That Flies Like and Airplane and Hovers Like a Helicopter". Proc. 2005 IEEE/ASME International Conference on Advanced Intelligent Mechatronics. 24-28 July Monterey, California.

11 A. Frank, M. Valenti, D. Leveine, J. How. " Hover Transitions and Level Flight Control Design for a SinglePropeller Indoor Airplane", Proc. AIAA Guidance Navigation and Control conference and Exhitbit, August 2007, Hilton Head South Carolina.

12 R. Cory and R. Tedrake. "Experiments in Fixed-Wing UAV Perching". Proc. AIAA Guidance Navigation and Control Conference, August 2008, Honolulu Hawaii.

13 B. Johnson and R. Lind, "Characterizing Wing Rock with Variations in Size and Configuration of Vertical Tail" AIAA Journal of aircraft, Vol. 47, No. 2, March-April 2010, pp 567 - 577.

14 D. Yeo, E. Atkins, W. Shyy, "Experimental Investigation of the Pressure, Forces and Torque Characteristics of a Rigid Flapping Wing ," Proc. 50 th AIAA Aerospace Sciences Meeting, January 2012, Nashville, Tennessee. (AIAA 2012-0849)

15 M. Patel, Z. Sowle, T. Corke and C. He, "Autonomous Sensing and Control of Wing Stall Using a smart Plasma Slat”, Proc. 44 ${ }^{\text {th }}$ AIAA Aerospace Sciences Meeting, 9-12 January 2006, Reno Nevada, (AIAA 2006-1207)

16 P.Bowles and T. Corke. "Stall Detection on a Leading-edge Plasma Actuated Pitching Airfoil Utilizaing Onboard Measurement", Proc. 47 $7^{\text {th }}$ Aerospace Sciences Meeting, 5-8 January 2009, Orlando Florida. (AIAA 2009-93)

17 C. Cox, A. Gopalarathnam, C. Hall. "Flight Test of Stable Automated Cruise Flap for an Adaptive Wing Aircraft". Journal of Aircraft, Vol. 47, No. 4, 2012 pp 1178-1188.

18 W. Barnwell directed by N. Chokani and C. Hall. "UAV Flight Control Using Distributed Actuation and Sensing". Masters Thesis, North Carolina State University 2003.

19 S. Lion directed by C.Hall. "Control Authorities of a distributed Actuation and Sensing Array on a BlendedWing-Body Uninhabited Aerial Vehicle". Masters Thesis, North Carolina State University 2007.

20 Air Force Office of Scientific Research (AFOSR) Multidisciplinary University Research Initiative (MURI) AFOSR Grant FA9550-05-1-0411, [online] http://www.avocet.gatech.edu

21 L. Schimdt. "Wing Rock Due to Aerodynamic Hysteresis", Journal of Aircraft, Vol. 16. No.3, March 1979. Pp129-133.

22 A. Anavatti, J. Choi and P. Wong, "Design and Implementation of Fuzzy Logic Controller for Wing Rock", International Journal of Control, Automation and Systems, Vol. 2, No. 4, December 2004. pp 494-500. 